\title{
Effects of Fast Acting Power Controller of BESS in the System Frequency Response of a Multi-Machine System: Probabilistic Approach
}

\author{
Francisco M. Gonzalez-Longatt, Senior Member IEEE \\ Centre for Renewable Energy Systems Technology - CREST \\ Loughborough University \\ Loughborough, United Kingdom \\ fglongatt@fglongatt.org
}

\begin{abstract}
This paper presents a probabilistic assessment of the effects of the fast acting power (FAP) controller of BESS in the system frequency response of a multi-machine system. Monte Carlo simulations and time-domain simulations, using DIgSILENT ${ }^{\circledR}$ PowerFactory ${ }^{\mathrm{TM}}$ are combined in the implementation of the proposed methodology. Simulation results show the effect fast-acting power controller of BESS in the system frequency response of a multi-machine system in the form of probabilistic distribution functions (PDF) of the main system frequency response.
\end{abstract}

Keywords-battery energy storage system, control system, frequency response; frequency, frequency response, inertia response.

\section{INTRODUCTION}

\section{A. Problem status and available solutions}

Ambitious targets in the volume of renewable generation have been defined in several countries around the world. However, the migration to a low carbon economy imposes several challenges to the economic an secure operation and control of the power systems [1]. One consequence on the increased integration of the low carbon technologies is the increased numbers of power electric converter-based devices, e.g. wind power, solar photovoltaic (PV) systems, electric vehicle charging stations, electricity storage systems, etc. The power electronic converter (PEC) tends to decouple the primary energy source from the synchronized power network. As a consequence, the share of synchronously connected power generation in the power system is decreasing, and it is decreasing the total locally available system's rotational inertia.

The secure and economical operation of low rotational inertia systems is a great challenge, especially because of the volatility of the system frequency. During a system frequency disturbance, the active power balance between the generation and demand is lost, there is a power imbalance $(\triangle P)$. Because the power balance is lost, the synchronous generators directedly connected to the power network will change the rotational speed $\left(\omega_{m e}\right)$, and the system frequency $(f)$ will change at a rate initially determined by the total system rotational inertia $\left(H_{T}\right)$ and the power imbalance $(\Delta P)$.

Lower volume of the total rotational inertia increases the likelihood of fast system changes and instability arising from progressively small disturbances that could lead to severe faults or loss of generation or demand [2]. It is expected a substantial decrease in the ability to overcome system frequency's disturbances, based on a decreased inertial response with overwhelming consequences for system frequency security and reliability [3], [4].

One possible solution to improve the system frequency response of low inertia rotational system is to enable the power electronic converter-based technologies with a novel controller that allow an active power sensible behaviour in response to changes in the system frequency. The idea of adding supplementary control loops to allow frequency response of the power converters has been extensively developed in the scientific literature, a summary of that method can be found in [5]. Several approaches propose been proposed in the literature for primary frequency response and may publications be dedicated to well-known inertia response, e.g. synthetic inertia, emulated inertia, releasing hidden inertia, fast power reserve emulation, etc. The inertia response controller has been successfully applied to several technologies like wind turbines [6], PV systems, battery energy storage systems (BESS) [7], etc.

The inertia controller must be recognised as a fast-acting active-power injection because the controller is designed to provide an injection/absorption of active power based on a proportional function of the rate of change of frequency (ROCOF). The inertia controller (independently of the implementation, and there are several in the scientific literature) tends to be recognised as a fast-acting controller because the speed response is proportional the ROCOF, and the last one has high values in low rotational inertia systems.

A methodology to probabilistically assess the effect of the inertial frequency response provided by BESS is introduced by F Gonzalez-Longatt et all in [8]. The methodology is illustrated by using a simple-machine system and considering the randomness provided by the power demand variability. However, Gonzalez-Longatt et all [8] has not included a 
compressive analysis of the multi-machine system. Also, the inertia controller used in that scientific paper is the most basic controller found in the literature.

An exhaustive assessment of the effects of the fast-acting power controller of BESS in the system frequency response of a multi-machine system is not available in the literature.

\section{B. Research objective and paper contributions}

This paper presents a probabilistic assessment of the effects of the fast-acting power controller of BESS in the system frequency response of a multi-machine system.

The paper unfolds the following contributions:

1. The concept of fast active power (FAP) controller is presented in this paper (see Section III). The FAP controller is a frequency sensible, and its response depends, mainly, on the rate of change of the local frequency.

2. A probabilistic assessment of the effects of FAP controller of BESS on the system frequency response is used to make evident the drawbacks of using the rate of change of the local frequency as dominant action in the FAP controller (see Section IV).

\section{Paper structure}

After the introduction in Section I, the paper has organised as the following: Section II presents the main aspects of BESS modelling. Section III is dedicated to the concept FAP controlled and show how it can be used enable frequency response in a BESS. Section IV shows the numerical results of simulations and discusses the impact of FAP controller on the system frequency response of a multi-machine test system. Finally, the advantages of this controller are discussed in Section V.

\section{MODELling OF BATTERy ENERGY STORAGE SYSTEM (BESS)}

Fig. 1 shows a generic model of a BESS; the model consists of three main sub-systems [6, 7]: (a) a power conversion system (PCS) (b) the battery energy system (BES), and (c) the controllers associated to the BESS.

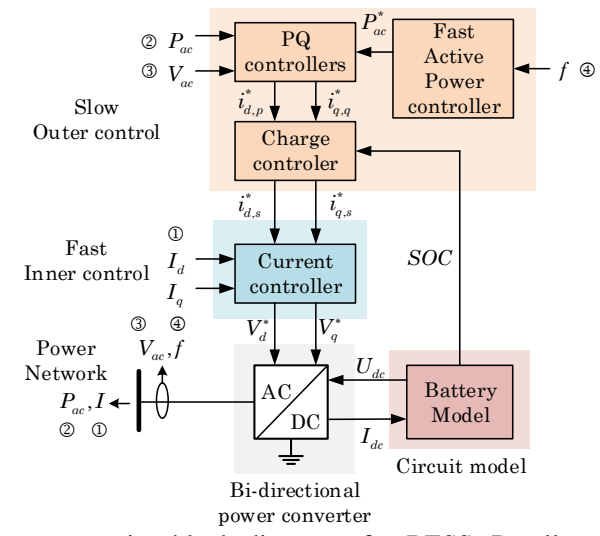

Fig. 1. A representative block diagram of a BESS. Details of the relevant variables and subsystem are shown.

This paper uses an improved version of the models published in [9]-[12]. A general overview of the main modelling aspects is presented in the next subsections. The full details of the FAP controller are presented in Section III. The BES uses a battery pack as the main element of store/release electricity and the electrochemical behaviour of the battery cell is interictally related to the battery technology. However, a simplified model can be used, and the model parameters can be adjusted to represent the battery technology. In this paper, the BES model is based on a simplified equivalent circuit; the series electrical circuit is used to model the electrical dynamic of the battery including the time dependence and the state-ofcharge (SOC). The SOC is calculated by an integrator which takes into account the current of the battery $\left(I_{\text {batt }}\right)$ :

$$
U_{d c}(t)=U_{\max } S O C(t)+U_{\max }[1-S O C(t)]-I_{\text {batt }}(t) R_{\text {in }}
$$

where $U_{\min }$ is the minimum voltage of the battery cell, also known as the voltage discharged cell in volts, $U_{\max }$ is the maximum voltage of the battery cell in volts, $I_{\text {batt }}$ is the current of the battery cell in amps.

The controllers used in the BESS can be devised in two main sets of controllers: inner controllers and outer controllers. The inner controller is a very fast controller, and it is related to the control of the $d q$-axis currents, and it is directly connected to the PEC. The outer controllers have slower time response, and they are related to more high-level actions: $P Q$-control, charge control, and FAP control (discussed in Section III).

Inner controller: Model of the current controller. A simple proportional-integral (PI) control is used for the $d$ and $q$-axis currents.

Model of the battery charge controller. It is designed to keep the $S O C$ inside boundary conditions $\left(S O C_{\min } \leq S O C \leq\right.$ $\left.S O C_{\max }\right)$. The charge controller uses the $d q$-axis currents as a control mechanism to restrict the charging or discharging of the BES.

Model of the PQ-Controller. The voltage (or $Q$ ) controller has a very slow current controller for set point tracking and a slope with a dead band for proportional voltage support.

\section{MODEL OF FAST ACTIVE POWER (FAP) CONTROLLER}

This section deals with the concept of fast active power (FAP) injection/absorption as a control strategy used to enable frequency responsive mode on power electronic converterbased technologies, e.g. generation/storage. The FAP controller is mainly characterised by a very quick response, typically defined by a very short time-delay (typically related to measurement rather than activation). There is not a universal definition of FAP at the moment but delivering full power in less than a second is used in this paper. Also, this paper presents the concept of FAP controller where the core of the control action is dominated by the rate-of-change-of-thelocal-frequency; there are few other controllers and proportional-limited, etc., but there are not discussed here,

Before embarking on a full discussion of the FAP control, it is important to have a clear understanding of the difference between the frequency response provided by the rotational inertial in synchronous generators and the FAP provided by 
power electronic converter-based technologies. The electromechanically dynamic behaviour of a synchronous generator immediately after a system frequency disturbance is a natural consequence of the physical design of the synchronous machine. The rotor of a synchronous generator has an inherent physical characteristic called inertia; it quantifies the tendency of the machine rotor to resist angular acceleration. The rotational inertia is inherent of synchronous generators directly connected to the power network; it provides natural and immediately damp disturbances to system frequency.

Several controllers have been defined in the literature in order to enable the frequency response of power electronic converter-based technologies. All of those controllers actuate on the active power reference $\left(P^{*}{ }_{a c}\right)$ of the power converter by including and increment/decrement that is a function of the locally measured frequency $(f)$. The wind turbine industry has explored and developed the concept of inertia response [12], it has several names: Artificial, Emulated, Simulated, or Synthetic Inertia. The inertia response concept allows a controller to the take the kinetic energy from the rotating mass in a wind turbine generator (WTG) [13]. The gain of the inertia controller $\left(H_{s y n}\right)$ has some physical meaning in the case WTG because the energy delivered to the power network is taken from the kinetic energy of rotational inertia. However, the gain of the inertia controller has not a direct interpretation in the case of non-rotating technologies, like PV, BESS, electric vehicle (EV) charger stations, etc. Some scientific papers as [7], [11] has applied the concept of inertia controller to BESS, but instead of taking kinetic energy from the rotating masses, the controller enables to discharge the battery in a controlled way producing an additional power in the form of inertial power $\left(\Delta P_{s y n}\right)$.

The synthetic inertia controller can be understood as a simple loop that increases the electric power output of the PCS during the initial stages of a significant downward frequency event. The inertial power or power produced during the system frequency disturbance is calculated using the equivalent to the swing equation of a synchronous generator [14]:

$$
\Delta P_{s y n}=2 H_{s y n} \frac{d f(t)}{d t}
$$

where $H_{s y n}$ represents the value of the synthetic inertia (sec) and $f$ is system frequency (p.u) and $\Delta P_{s y n}$ represent the socalled inertia power $\left(P_{a c}^{*}=\Delta P_{s y n}\right.$, see Fig. 1).

The control rule defined by (2) includes the use of the rateof-change-of-the-local-frequency, the derivative term provides a very fast response, but the compensation could violate physical limits (for instance, $d P / d t$ ) damaging the components (e.g. proving early fatigue braking in WTG). Also, the frequency measurements in a real power network have a reasonably high signal-to-noise ratio; as a consequence, the derivative control amplifies the noise level imposing excessive stress on the generation/storage technology.

Fig. 2 shows a fast-active-power (FAC) controller which is implemented using a derivative controller and compensated by a first order low-pass filter and the output signal is limited. The discussion of the controller properties from the control theory point of view is beyond the scope of this paper, but next sections partially evaluate the impact of the parameter selection in the system frequency response of the power network.

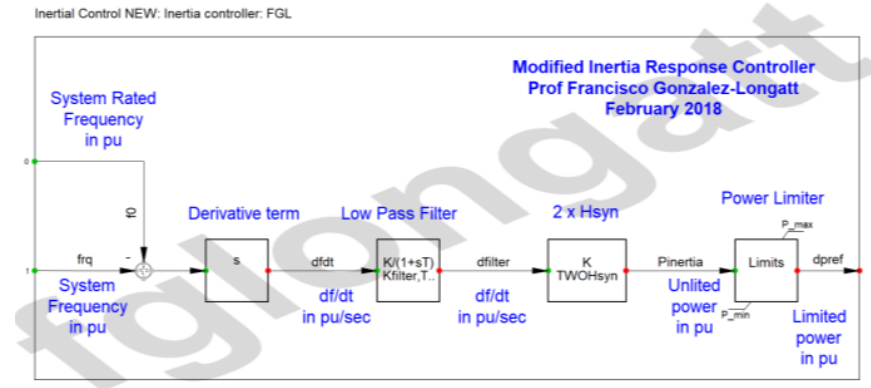

Fig. 2. Implementation of a fast-active-power (FAP) controller.

The FAP controller is designed to exchange (absorb or inject) active power between the generation/storage unit and the power network, but this controller does not contribute to the system rotational inertia of the power network. One important consideration in the FAP controller is the quality of the measurement of the power network variables, the measurement process takes times which adds delay to the control action, and the first-order low pass filter also adds control delay in the response. Considering all the time delays of measurement and control the FAP control has the possibility of delivering the full power of the assess in less than 1.0 second.

\section{Probabilistic Assessment of System FrequenCy RESPONSE: SIMULATION AND RESULTS}

\section{A. System Frequency Response Indicators}

Several performance indicators are available in the scientific literature to describe and to evaluate the system frequency response (SFR). In this paper, the FAP controller is designed to affect the frequency response during a short time; the main interest is located sub-primary frequency response. Three classical SFR indicators are considered in this paper: (i) Minimum frequency or frequency nadir ( $f_{\text {min }}$, unit: Hertz) measures the minimum point of the time-domain response of system frequency after the system frequency disturbance. (ii) Steady-state frequency ( $f_{s s}$, units: Hz). Quasi-steady-state frequency is the final value of the system frequency after the system frequency disturbance. (iii) Maximum initial frequency gradient ([df/dt $]_{t=0+}$, unit: Hertz/seconds), it represents the maximum rate-of-change-of-frequency immediately after the system frequency disturbance $\left(t=0^{+}\right)$, and as observed by ROCOF relays.

The concept of centre of inertia (COI) is used to represent the system frequency assessment of a multi-machine system. The frequency of the COI $\left(f_{\mathrm{COI}}\right)$ corresponds to the inertiaweighted average of all generator frequencies: 


$$
f_{\text {COI }}=\frac{\sum_{k=1}^{N_{g}} H_{k} f_{k}}{\sum_{k=1}^{N_{g}} H_{k}}
$$

where $N_{g}$ is the total number of synchronous generators directly connected to the power network, is the total inertia of the system, $H_{k}$ and $f_{k}$ are the rotational inertia and the electrical frequency of the $k$-th generator, respectively.

\section{B. Methodology of the Probabilistic Assessment}

This paper uses the methodology presented in [8], it consists of the following sequence of four steps: (i) Uncertainties modelling, (ii) Scenarios creation, (iii) Monte Carlo Simulation, (iv) Probabilistic analysis of the results.

This paper considers the uncertainties of the loads, as a consequence power is modelled as a random variable $\left(P_{L, i}\right)$ normally distributed within each hour for a given time period [17]. Then, the probability distribution function (PDF) of the power demand of the $i$-th load $\left(P_{L, i}\right)$ is given by the following expression:

$$
f_{P_{L, i}}\left(P_{L, i}\right)=\frac{1}{\sigma_{i} \sqrt{2 \pi}} e^{-\frac{\left(P_{L, i}-\mu_{L, i}\right)^{2}}{2 \sigma_{i}^{2}}}
$$

where $\mu_{L, i}$ is the mean value of the electric demand and $\sigma_{i}$ is the standard deviation.

A set of simulation scenarios are created considering the power demand randomness, then Monte Carlo simulations (MSC) for each scenario are performed, time-domain numerical simulations are used to produce the main variables representing the system frequency response of each scenario. Finally, the time domain series are post-processed to obtain the main system frequency indicators and creating the correspondent PDF's.

\section{Simulation AND Results}

\section{A. Test System}

The classical WSCC 3-machine, 9-bus system is used for the system frequency assessment purposes in this paper. The test system has been modified to integrate three BESS (50MW, rated power each) in bus 4,7 and 9 (see Fig. 7), power dispatch is set at $P_{G 2}=163.0 \mathrm{MW}$ and $P_{G 3}=85 \mathrm{MW}$, the generation units are equipped with governors and exciting. The test network all generator unit controllers are developed using DIgSILENT ${ }^{\circledR}$ PowerFactory ${ }^{\mathrm{TM}} 2018$ SP1, and the model of the BESS are created using DigSILENT Simulation Language (DSL) considering the models and parameters shown in the previous sections, the BESS has been enabled to provide system frequency response using the proposed FAP controller.

\section{B. Load demand uncertainty}

For illustrative purposes, a single source of power system uncertainty is considered in this paper. The uncertainties coming from the demand are modelled using a Gaussian probabilistic distribution: $P_{\text {loadA }}\left(\mu_{\text {PloadA }}=125.0 \mathrm{MW}\right), P_{\text {loadB }}$ $\left(\mu_{\text {PloadB }}=90 \mathrm{MW}\right), P_{\text {loadC }}\left(\mu_{\text {Pload }}=100 \mathrm{MW}\right)$, all loads are assumed to have the same standard deviation $\left(\sigma_{\text {PloadA }}=\sigma_{\text {PloadB }}\right.$ $=\sigma_{\text {PloadC }}= \pm 50 \%$ ) and there is no correlation considered (see Fig. 4). A preliminary analysis of the SFR considers the $N-1$ contingency based on sudden disconnection of a single generator in the test system. Fig 5 shows the time-domain plots of $f_{C O I}(\mathrm{~Hz})$, and details of the minimum frequency are highlighted; CASE II sudden disconnection of $\mathrm{G} 2$ produces the deepest frequency deviation $\left(f_{\min }=56.88 \mathrm{~Hz}\right)$.

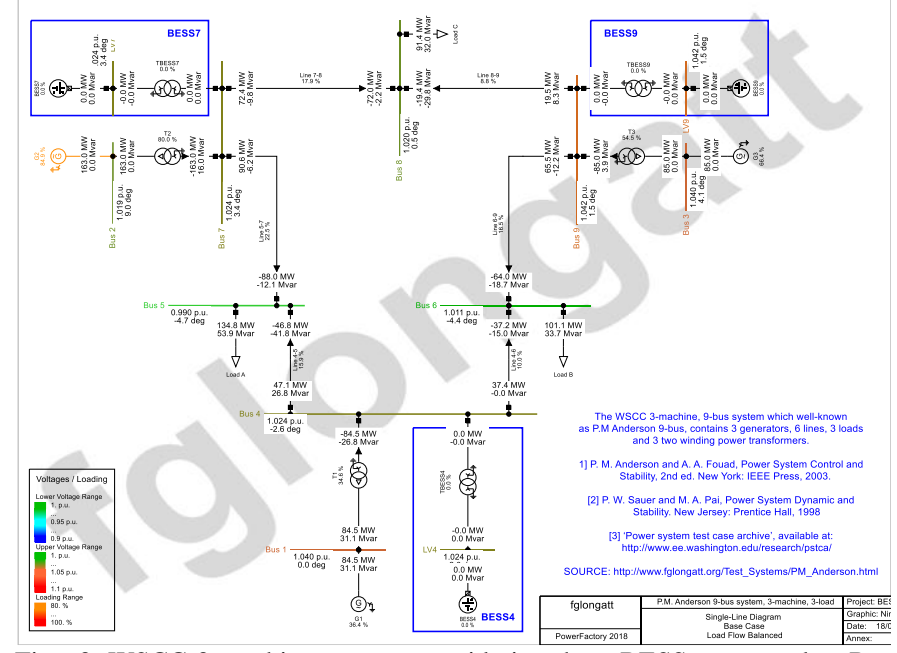

Fig. 3. WSCC 3-machine system, considering three BESS connected to Bus 4, 7 and 9. Also known as P.M Anderson 9-bus.

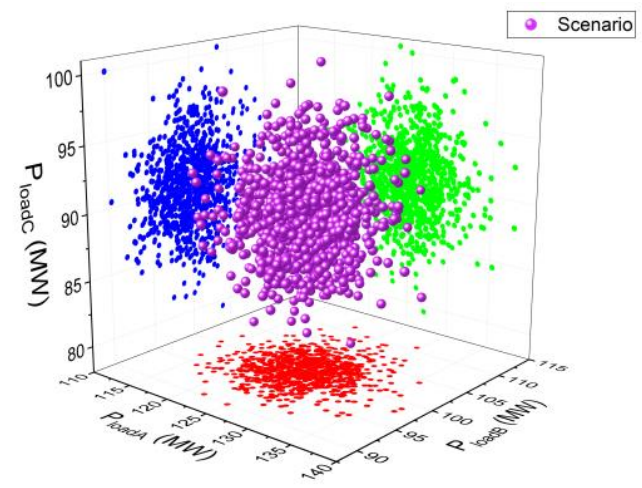

Fig. 4. Representation of the simulation scenarios, each axis represents the corresponding power demand $\left(N_{\text {samples }}=1,000\right)$.

\section{Base Case: Effect of Spinning reserve and disturbance location}

Fig 5 shows details of the frequency in each generator considering the sudden disconnection of G3. The effect of the spinning reserve on the SFR is based on three scenarios: High, Mid, and Low. As expected, high spinning reserve produces smaller frequency deviations and high minimum frequency.

A summary of the numerical results of the minimum frequency of centre of inertia $\left(f_{\mathrm{COI}}\right)$ and maximum rate of change of frequency of inertia centre $\left(d f_{\mathrm{COI}} / d t\right)$ for several cases of spinning reserve and generator outage are presented in Table I. 


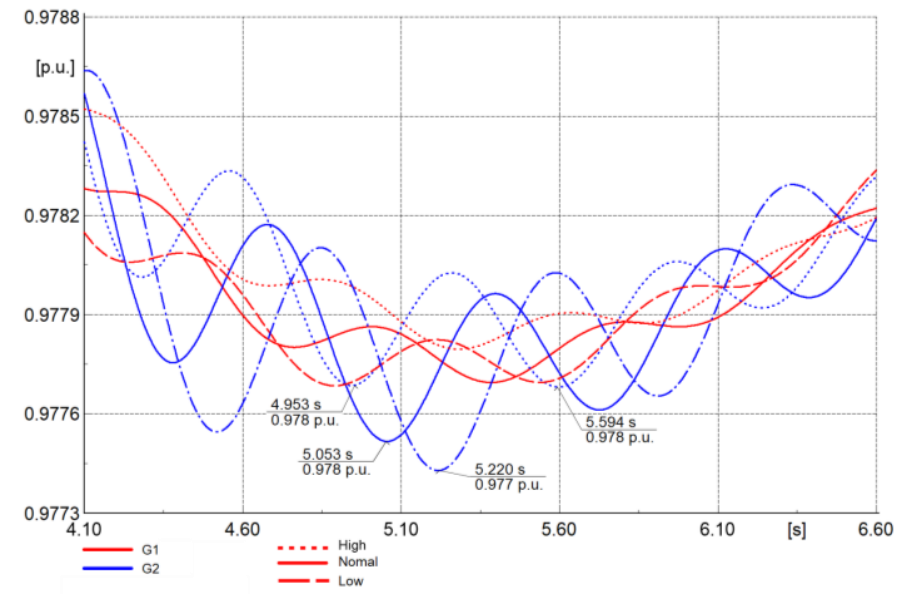

Fig. 5. Details of the electrical frequency (p.u) of G1 and G2, showing the minimum frequency. CASE III. G3 outage. Base Case: WITHOUT-BESS.

TABLE I. SUMMARY OF FREQUENCY RESPONSE INDICATORS: NO BESS

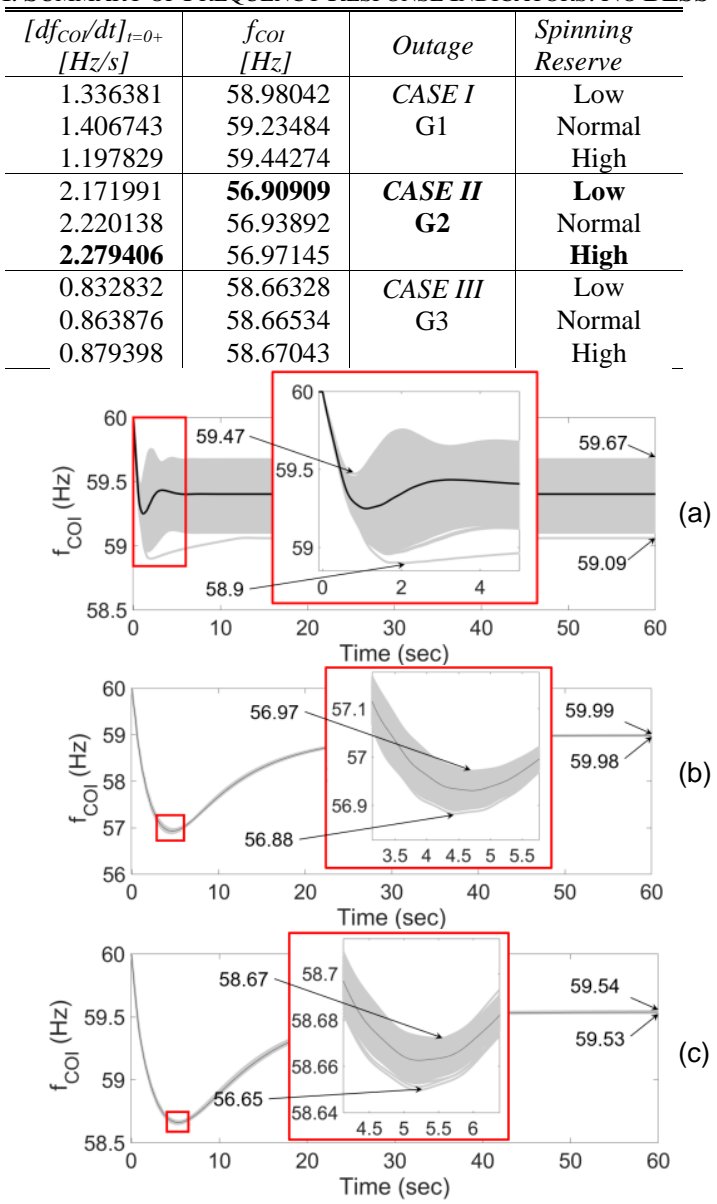

Fig. 6. Time-domain response of the frequency of COI, $f_{C O I}(\mathrm{~Hz})$. WITHOUTBESS. CASE I: G1 outage, CASE II: G2 ( $\triangle P=163 \mathrm{MW})$ outage CASE III: G3 outage $(\triangle P=85 \mathrm{MW})$. Simulation scenarios shown in Fig. 4.

The sudden disconnection of G2 (CASE II) produces the worst system frequency disturbance $(\triangle P=163 \mathrm{MW})$, low spinning reserve produces a deeper frequency exclusion, and high spinning reserve tends to increase the $\left[d f_{C O I} / d t\right]_{\mathrm{t}=0+}$. The time-domain plots $(1,000)$ of $f_{C O I}$ for the test system without BESS are shown in Fig. 6. The system frequency response follows the classical fish-hook "shape", and the main frequency indicators of the SFR are depicted in Fig. 7 $\left(\left[d f_{C O I} / d t\right]_{\mathrm{t}=0+}, f_{\min }\right.$, and $\left.f_{s s}\right)$. The CASE II produces the worstcase scenario with the deepest frequency excursion $\left(f_{\min }=\right.$ $56.88 \mathrm{~Hz})$ and ROCOF $\left([d f C O I / d t]_{t=0+}=2.30 \mathrm{~Hz} / \mathrm{sec}\right)$.

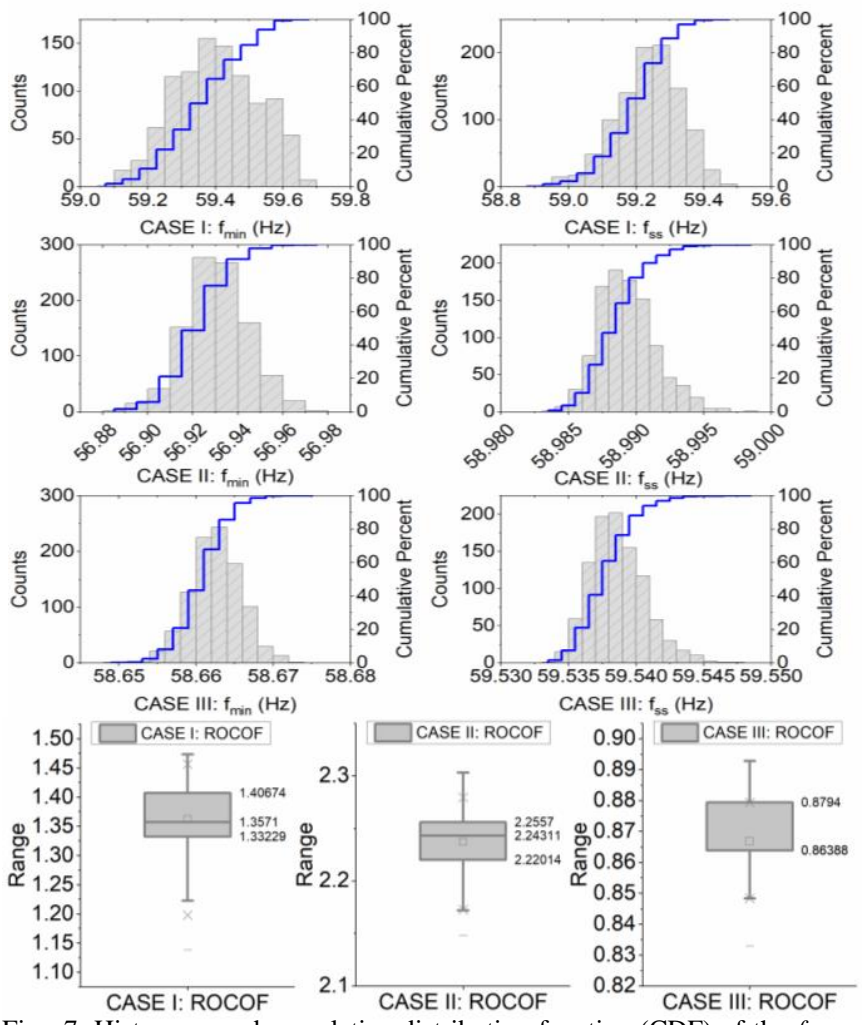

Fig. 7. Histogram and cumulative distribution function (CDF) of the $f_{\min }$ and $f_{s s} ;$ and boxplot of ROCOF (Hz/s). Base Case: WITHOUT-BESS.

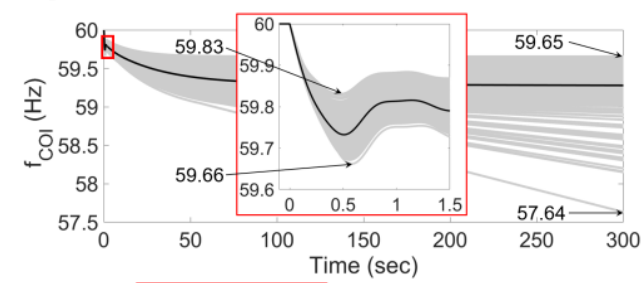

(a)
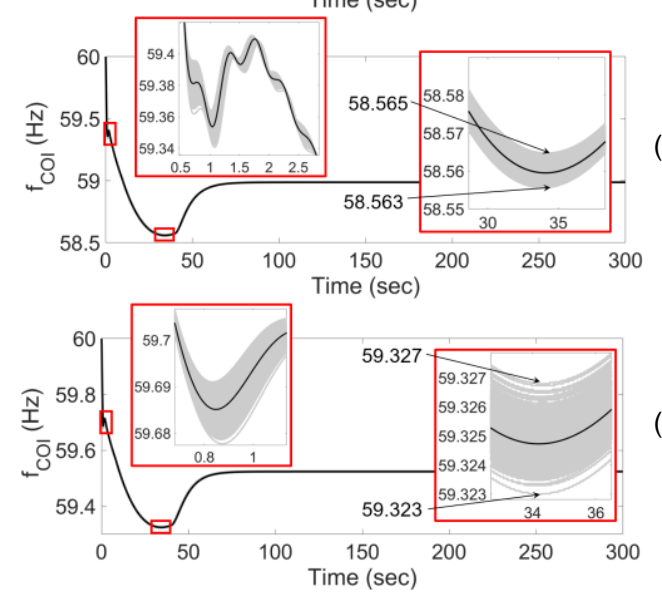

Fig. 8. Time-domain response of the frequency of COI, $f_{C O I}(\mathrm{~Hz})$. WITH BESS. CASE I: G1 outage, CASE II: G2 $(\triangle P=163 \mathrm{MW})$ outage CASE III: G3 outage $(\triangle P=85 \mathrm{MW})$. Simulation scenarios shown in Fig. 4 .

Now, three-BESSs are installed in the test system and the 
frequency response is analysed, Fig. 8 show the time-domain plot of the frequency of COI and relevant values are indicated. The SFR of the system is more complex in shape, in fact, there is an initial "small fish-hook" very short time after the frequency disturbance caused by the accelerated inertial power injected by the BESSs, and there is a second big fish-hook shape later in the SFR, it is caused by the slower electromechanical response of the generator controllers. As a consequence, a negative effect of the FAP controller is making SFR more complex including very fast transients (compared with the traditional electromechanical one). As the FAP controller is designed to provide an inertial response, the boxplot of ROCOF (Hz/s) is plotted in Fig 9. Discussions of $f_{\min }$ and $f_{s s}$ are not included in this paper for space considerations and other complexities no relevant to this paper.

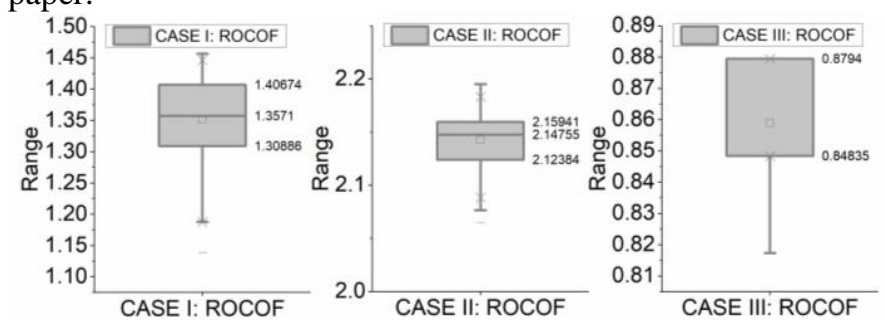

Fig. 9. Histogram and cumulative distribution function (CDF) of the $f_{\min }$ and $f_{s s} ;$ and boxplot of ROCOF (Hz/s). WITH-BESS.

The most positive effect of FAC controller is the reduction of the $[d f C O I / d t]_{t=0+}$, comparing the numerical results in Fig. 7 and 9, the ROCOF in the worst scenario (CASE II) of ROCOF passes from $2.30311 \mathrm{~Hz} / \mathrm{sec}$ to $2.19497 \mathrm{~Hz} / \mathrm{sec}$, and the average improvement in the ROCOF is between $0.7 \%$ to $4.7 \%$.

\section{CONCLUSIONS}

The concept of fast active power (FAP) controller is presented in this paper; it is a sensible frequency controller acting on the active power injection in a sub-second time scale. This paper uses a FAP controller which response depends, mainly, on the rate of change of the local frequency. This paper presents a probabilistic assessment of the effects of the FAP controller of BESS in the system frequency response of a multi-machine system. The methodology uses a combination of Monte Carlo simulations and time-domain simulations, using DIgSILENT ${ }^{\circledR}$ PowerFactory $^{\text {TM }}$. Simulation results show the effect fast acting power controller of BESS in the system frequency response of a multi-machine system in the form of probabilistic distribution functions (PDF) of the main system frequency response. The probabilistic assessment of the effects of FAP controller of BESS on the system frequency response is used to make evident the positive and negative aspects of using the rate of change of the local frequency as dominant action in the FAP controller: (i) introduces a very fast dynamic in the frequency helping to improve the system frequency response in the sub-second region, reducing the ROCOF, (ii) the finite amount of energy in the BESS combined with the inertial action changes the shape of the system frequency response creating a so called "double fish-hook" shape, this new shape requires further analysis. Especially in the effect of the FAP controller in the mechanical component of the generation units

\section{REFERENCES}

[1] F. Gonzalez-longatt and L. Rueda, "Introduction to Smart Grid Functionalities," in Advanced Smart Grid Functionalities Based on PowerFactory, 2018, pp. 1-18.

[2] A. Ulbig, T. S. Borsche, and G. Andersson, "Impact of Low Rotational Inertia on Power System Stability and Operation," IFAC Proc. Vol., vol. 47, no. 3, pp. 7290-7297, Jan. 2014.

[3] A. Bonfiglio, F. Delfino, F. Gonzalez-Longatt, and R. Procopio, "Steadystate assessments of PMSGs in wind generating units," Int. J. Electr. Power Energy Syst., vol. 90, pp. 87-93, 2017.

[4] M. Deepak, R. J. Abraham, F. M. Gonzalez-Longatt, D. M. Greenwood, and H.-S. Rajamani, "A novel approach to frequency support in a wind integrated power system," Renew. Energy, vol. 108, pp. 194-206, 2017.

[5] F. Gonzalez-Longatt, Frequency control and inertial response schemes for the future power networks. 2014.

[6] H. R. Chamorro, N. R. Malik, F. Gonzalez-Longatt, and V. K. Sood, "Evaluation of the synthetic inertia control using active damping method," in 2017 6th International Conference on Clean Electrical Power (ICCEP), 2017, pp. 269-274.

[7] F. M. Gonzalez-Longatt and S. M. Alhejaj, "Enabling inertial response in utility-scale battery energy storage system," in IEEE PES Innovative Smart Grid Technologies Conference Europe, 2016.

[8] F. Gonzalez-Longatt, S. Alhejaj, A. Bonfiglio, R. Procopio, and J. L. Rueda, "Inertial frequency response provided by battery energy storage systems: Probabilistic assessment," in 2017 6th International Conference on Clean Electrical Power: Renewable Energy Resources Impact, ICCEP 2017, 2017, pp. 403-409.

[9] F. M. Gonzalez-Longatt and S. M. Alhejaj, "Enabling inertial response in utility-scale battery energy storage system," in 2016 IEEE Innovative Smart Grid Technologies - Asia (ISGT-Asia), 2016, pp. 605-610.

[10]S. M. Alhejaj and F. M. Gonzalez-Longatt, "Impact of inertia emulation control of grid-scale BESS on power system frequency response," in 2016 International Conference for Students on Applied Engineering (ICSAE), 2016, pp. 254-258.

[11]S. M. Alhejaj and F. M. Gonzalez-Longatt, "Investigation on grid-scale BESS providing inertial response support," in 2016 IEEE International Conference on Power System Technology, POWERCON 2016, 2016.

[12]F. Gonzalez-Longatt, S. Alhejaj, A. Bonfiglio, R. Procopio, and J. L. Rueda, "Inertial frequency response provided by battery energy storage systems: Probabilistic assessment," 2017 6th Int. Conf. Clean Electr. Power Renew. Energy Resour. Impact, ICCEP 2017, pp. 403-409, 2017. 